ARTICLE

DOI: $10.1038 / s 41467-017-00241-z$

\title{
Hole-phonon coupling effect on the band dispersion of organic molecular semiconductors
}

\author{
F. Bussolotti ${ }^{1,4}$, J. Yang ${ }^{2,5}$, T. Yamaguchi ${ }^{1,3}$, K. Yonezawa $^{1}$, K. Sato ${ }^{2}$, M. Matsunami ${ }^{1,6}$, K. Tanaka ${ }^{1,3}$, \\ Y. Nakayama ${ }^{2,7}$, H. Ishii ${ }^{2}$, N. Ueno ${ }^{2} \&$ S. Kera ${ }^{1,2,3}$
}

The dynamic interaction between the traveling charges and the molecular vibrations is critical for the charge transport in organic semiconductors. However, a direct evidence of the expected impact of the charge-phonon coupling on the band dispersion of organic semiconductors is yet to be provided. Here, we report on the electronic properties of rubrene single crystal as investigated by angle resolved ultraviolet photoelectron spectroscopy. A gap opening and kink-like features in the rubrene electronic band dispersion are observed. In particular, the latter results in a large enhancement of the hole effective mass $(>1.4)$, well above the limit of the theoretical estimations. The results are consistent with the expected modifications of the band structures in organic semiconductors as introduced by hole-phonon coupling effects and represent an important experimental step toward the understanding of the charge localization phenomena in organic materials.

\footnotetext{
${ }^{1}$ Institute for Molecular Science, Myodaiji, Okazaki 444-8585, Japan. ${ }^{2}$ Graduate School of Advanced Integration Science, Chiba University, Chiba 263-8522, Japan. ${ }^{3}$ SOKENDAI (The Graduate University for Advanced Studies), Hayama, Kanagawa 240-0193, Japan. ${ }^{4}$ Present address: Institute of Materials Research and Engineering (IMRE), 2 Fusionopolis Way, Innovis, Singapore \#08-03, Singapore. ${ }^{5}$ Present address: College of Physical Science and Technology, Yangzhou University, Jiangsu 225009, People's Republic of China. ${ }^{6}$ Present address: Toyota Technological Institute, 2-12-1 Hisakata, Tempaku-ku, Nagoya 468-8511, Japan. ${ }^{7}$ Present address: Department of Pure and Applied Chemistry, Faculty of Science and Technology, Tokyo University of Science, 2641 Yamazaki, Noda-shi, Chiba-ken 278-8510, Japan. Correspondence and requests for materials should be addressed to F.B. (email: b.fabio@imre.a-star.edu.sg) or to S.K. (email: kera@ims.ac.jp)
} 
$\mathrm{n}$ recent years, the use of organic molecular semiconductors as base materials for optoelectronic applications marked a significant advancement in the technological field, resulting in an entirely new class of light, flexible and low-cost devices ${ }^{1-3}$. Despite the technological achievements, important aspects of the physics of these materials remain still elusive, as for example the exact nature of the charge transport mechanism. This important issue was originally addressed by applying concepts and ideas developed in transport studies of inorganic semiconductors and metals. In this context, the charge transport in organic molecular semiconductors was described with respect to the limiting case of coherent band-like transport and incoherent hopping-like transport $^{4}$ corresponding to the extreme delocalization and localization of the charge carriers, respectively. The former mechanism is dominated by the energy dispersion of the highest occupied (HOMO) and lowest unoccupied (LUMO) molecular orbital derived bands which reflects the spatial overlap of the molecular wavefunctions ${ }^{4}$. The latter mechanism is affected by the charge reorganization energy which depends on the hole/ electron coupling with the intramolecular vibrations, as molecules generally undergoes structural relaxations upon receiving additional charges ${ }^{4,5}$. However, both the conventional band and hopping transport theories soon appeared inadequate for describing the measured charge mobility in a large number of organic materials ${ }^{6}$. As a matter of fact, the charge localization/ delocalization phenomena in molecular organic semiconductors occur on comparable characteristic energy scale $\left(\sim 50-200 \mathrm{meV}^{4}\right)$ which prevents the applicability of the above mentioned conventional limiting treatments ${ }^{6}$. More realistically, the charge transport mechanism in molecular organic semiconductors is expected to result from the delicate interplay between charge spatial delocalization vs. localization processes ${ }^{6-8}$ both affected, in turn, by the details of the crystal structure and temperature $e^{4,6-8}$. Intense theoretical research is still on-going to include all these critical aspects in a fully consistent and accurate description of the charge carrier dynamics in organic molecular semiconductors ${ }^{6-8}$. More recently, the hole/electron coupling with intermolecular vibrations was also discussed as a possible origin of charge localization, as related to the continuous modulation of the spatial overlap between molecular wavefunctions ${ }^{7-10}$.

In this context, experimental studies on the impact of the charge-phonon coupling on the electronic properties of the organic molecular semiconductors may provide critical information (i.e., coupling strength, phonon energies, and so on) for refining the details of the various transport models and/or testing the validity of the corresponding predictions.

In metals, the charge coupling with lattice phonons manifests in local change in the curvature of the band dispersion (i.e., kink structures), as directly revealed by a number of angle resolved ultraviolet photoemission spectroscopy (ARUPS) investigations ${ }^{9}$. The kink position and the degree of the related band distortion reflect the energy of the phonons and the strength of their coupling with the charge carriers. These effects may result, for example, in the enhancement of the carrier effective mass at the Fermi level, with consequent impact on the charge mobility ${ }^{9}$. Local change in the band curvature was similarly predicted to appear in the band dispersion of organic semiconductors, originating from the charge coupling with molecular vibrations ${ }^{11}$. However, despite theoretical predictions and relevance for applications, a direct experimental evidence of band distortions in organic molecular semiconductors as mediated by the charge-phonon coupling has yet to be observed.

In this paper, we report on the ARUPS investigation of the band dispersion of rubrene single crystal. With one of the highest reported hole mobility value $\left(\sim 40 \mathrm{~cm}^{2} / \mathrm{Vs}\right)^{12}$ among molecular organic semiconductors, rubrene single crystal represents a prototype system for both fundamental and applicative studies $^{6-8,12-15}$. A gap opening and kink-like distortion of the HOMO derived band dispersion are revealed. The results are consistent with the expected modifications of the band structures in organic semiconductor as introduced by hole-phonon coupling effects, as predicted by theoretical calculations on organic models system $^{11}$. This result directly confirms the leading role of molecular vibrations in affecting the charge localization in organic single crystals, which is critical for charge mobility ${ }^{6-8}, 12-15$. a

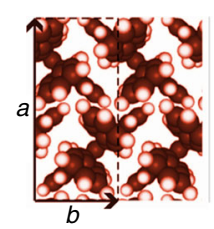

b
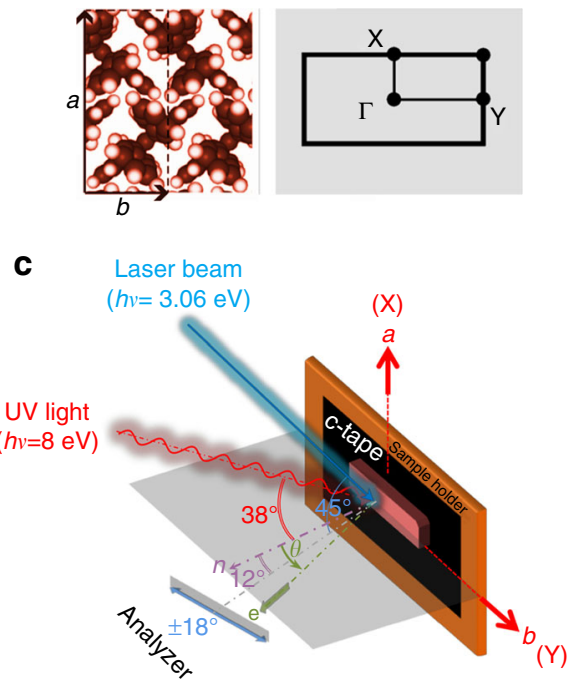

d

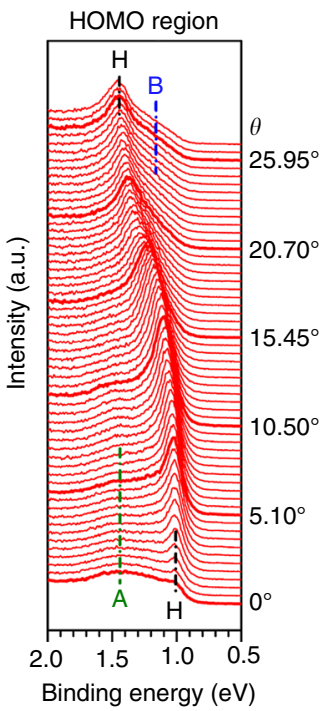

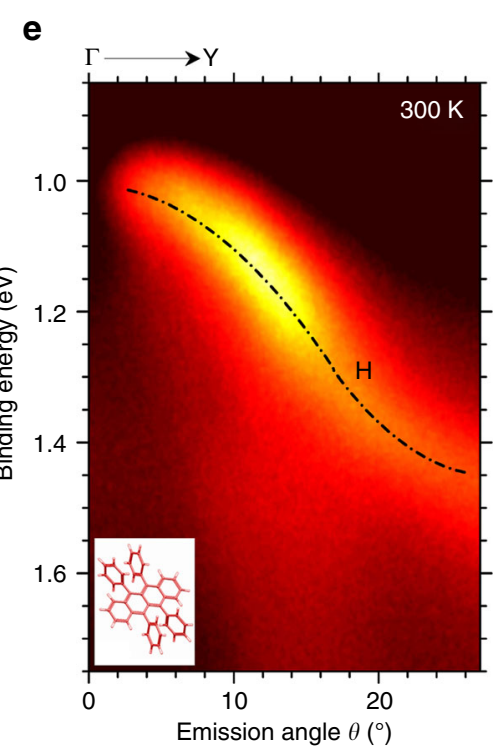

Fig. 1 Crystal structure with ARUPS experimental geometry and data of rubrene single crystal. Schematic of the molecular orientation in the crystalline $a-b$ plane of rubrene single crystal $(a=14.4 \AA, b=7.2 \AA$ ) in real $\mathbf{a}$ and reciprocal space $\mathbf{b}$. c Schematic drawing of the experimental geometry. $\mathbf{d} \theta$ dependence of the ARUPS spectra of rubrene at $300 \mathrm{~K}$ along the $\Gamma Y$ direction. The peak positions of the HOMO $(\mathrm{H})$ and of non-dispersive features $\mathbf{a}$ and $\mathbf{b}$ are indicated by dash dotted vertical lines (see text for further details). e ARUPS spectra intensity as a function of the emission angle $\theta$ along the $\Gamma Y$ direction. The HOMO $(\mathrm{H})$ dispersions is indicated by dash dotted black curve as guides for the eye. A sketch of the rubrene molecule is reported in the inset for reference 
a

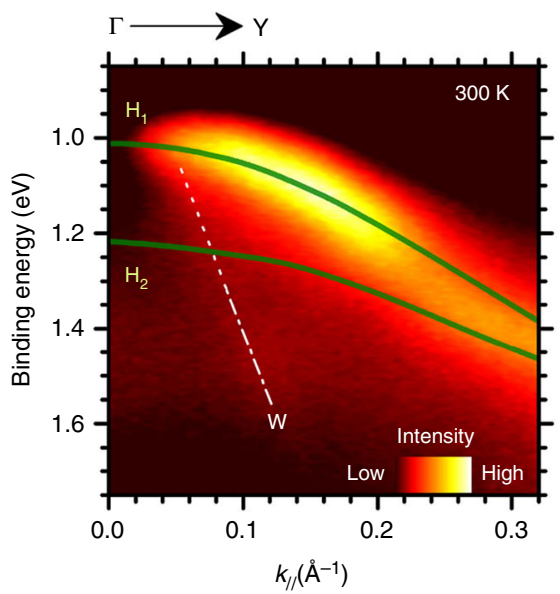

b

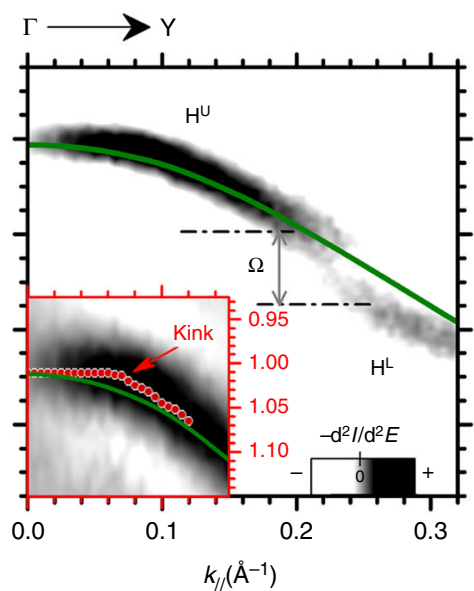

c

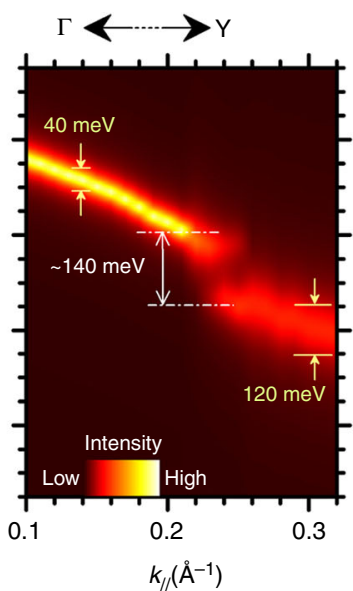

Fig. 2 Angle resolved band map at $300 \mathrm{~K}$ and theoretical calculations. a ARUPS intensities vs. $k / /$ along the $\Gamma Y$ direction of rubrene single crystal at $300 \mathrm{~K}$. Theoretical HOMO band dispersions are plotted as continuous green lines. $\mathbf{b}$ Second derivative ARUPS intensity map (- $\mathrm{d}^{2} \mathrm{I} / \mathrm{d}^{2} E$, where $I$ is the ARPES intensity and $E$ the binding energy) as obtained from data in $\mathbf{a}$. The splitting of the $\mathrm{HOMO}$ band in two subband $\mathrm{H}^{\mathrm{U}}$ and $\mathrm{H}^{\mathrm{L}}$ is evidenced as separated by gap $\Omega$. Inset: Magnification of second derivative map close to $\Gamma$ point. Experimental $H$ peak positions are also indicated by red circles and compared with theoretical band dispersion (continuous green line) to highlight the kink-like distortion. c Intensity map of $\mathrm{H}$ peak Lorentzian component along the $\Gamma Y$ direction, as extracted from HOMO peak fitting procedure. The intensities of Lorentzian components were normalized with respect to the peak area

\section{Results}

ARUPS measurements of the HOMO band dispersion. The rubrene crystal structure in real and reciprocal space are described in Fig. 1a, b, respectively, while the ARUPS experimental geometry is schematically depicted in Fig. 1c.

Figure 1d shows the $\theta$ dependence of the ARUPS spectra in the HOMO binding energy region of a rubrene single crystal, as acquired at $300 \mathrm{~K}$ along the $\Gamma Y$ high symmetry direction (Fig. 1b). In real space, the $\Gamma Y$ direction corresponds to the $b$ axis (Fig. 1a) of the rubrene unit cell along which the highest hole mobility values were reported ${ }^{12,14,15}$. The corresponding ARUPS intensity map is shown in Fig. 1e. In the ARUPS spectra a peak like, energy dispersive feature $(\mathrm{H})$ is clearly visible [Fig. 1d, e]. At low emission angle $\left(\theta \leq 5^{\circ}\right)$ the intensity of the $H$ peak rapidly decreases near the $\Gamma$ point $\left(\theta=0^{\circ}\right)$. The attenuation can be qualitatively explained in terms of photoemission matrix effect, reflecting the UV light polarization and the symmetry of the initial (HOMO) and final (photoelectron) states as coupled by the dipole transition ${ }^{16}$. This effect was previously reported to modulate the intensity of the ARUPS spectra of crystalline materials around the high symmetry points of the reciprocal space $^{17}$. Close to the $\Gamma$ point a broad, non-dispersive spectral feature (A) is also observed, as centered at $\sim 1.4 \mathrm{eV}$ of binding energy. At higher emission angle $\left(\theta>20^{\circ}\right)$ a shoulder (B) appears at $\sim 1.25 \mathrm{eV}$, located on the low binding energy tail of the HOMO peak $\mathrm{H}$.

Rubrene HOMO band dispersion at $300 \mathrm{~K}$. The HOMOARUPS intensity map as a function of the in-plane wave vector component $\left(k_{/ /}\right)$along $\Gamma \Upsilon$ is reported in Fig. 2a. The calculated HOMO bands [as extracted from ref. ${ }^{18}$ ] are overlaid as continuous lines to the experimental data. Two HOMO derived bands $\left(\mathrm{H}_{1}\right.$ and $\left.\mathrm{H}_{2}\right)$ were theoretically predicted ${ }^{18}$. The HOMO band splitting originates by the presence of two structurally inequivalent molecules in the in-plane rubrene unit cell (Fig. 1a) ${ }^{18}$ as similarly reported for other organic molecular semiconductors ${ }^{4}$.

The calculated $\mathrm{H}_{1}$ band reproduces quite well the $\mathrm{H}$ band dispersion, while no clear experimental evidence of $\mathrm{H}_{2}$ band is found. Moreover, the presence of $\mathrm{A}$ and $\mathrm{B}$ feature is not supported by the theoretical calculations. These differences are discussed in Supplementary Note 1 as side aspects of the hole-vibration coupling mechanism demonstrated in the present work (Supplementary Fig. 1a, b).

Finally, a rapidly dispersive $w$ band is observed, which was assigned to photoelectron scattering into unoccupied states of rubrene single crystal. A detailed discussion on these effects is included in Supplementary Note 2 with the support of the experimental data reported in Supplementary Figs 2-4.

While the overall behavior of $\mathrm{H}$ band dispersion is quite consistent with the theoretical calculations, small but finite differences exist as revealed by the second derivative analysis of the ARUPS data (Fig. $2 \mathrm{~b}$ and inset). In particular, a gap-like structure is observed at $\sim 0.22 \AA^{-1}$ with the $\mathrm{H}$ band splitted into an "upper" $\left(\mathrm{H}^{\mathrm{U}}\right)$ and "lower" $\left(\mathrm{H}^{\mathrm{L}}\right)$ subband (Fig. $2 \mathrm{~b}$ ) and a kink-like feature is observed in the experimental band dispersion near the $\Gamma$ point (inset of Fig. $2 b$ ).

The HOMO energy distribution curves (EDCs) at different $k_{/ /}$values were analyzed by least square peak fitting procedure, with the $\mathrm{H}$ peak simulated by a Voigt function according to the procedure described in Methods section. The as-extracted Lorentzian components of $\mathrm{H}$ peak, which reflects the hole quasiparticle dynamics ${ }^{16}$, were reported at variance of $k_{/ /}$in the color intensity map of Fig. 2c (EDC fitting analysis in Methods section). A value of $\Omega=140 \pm 20 \mathrm{meV}$ was estimated from the $\mathrm{H}^{\mathrm{U}}$ and $\mathrm{H}^{\mathrm{L}}$ peak separation in the $\left[0.20 \sim 0.22 \AA^{-1}\right]$ wave vector range. Upon crossing the energy gap, the Lorentzian full width at half maximum $\left(w_{\mathrm{L}}\right)$ increases from $40 \mathrm{meV}$ $\left(\mathrm{H}^{\mathrm{U}}\right.$ band) to $120 \mathrm{meV}$ ( $\mathrm{H}^{\mathrm{L}}$ band) (Fig. $2 \mathrm{c}$ ) which corresponds to a decrease of the hole lifetime $\tau\left(=\hbar / w_{\mathrm{L}}{ }^{14}\right)$.

The data reported in Fig. $2 \mathrm{~b}$ may suggest a possible identification of the $\mathrm{H}^{\mathrm{U}}$ and $\mathrm{H}^{\mathrm{L}}$ subbands with $\mathrm{H}_{1}$ and $\mathrm{H}_{2}$ theoretical band, respectively, their intensity being suddenly modulated by photoemission matrix element effect. This possibility is excluded on the basis of the theoretical and experimental arguments presented in Supplementary Note 1.

Rubrene HOMO band dispersion at $110 \mathrm{~K}$. ARUPS measurements were repeated upon cooling rubrene single crystal to $110 \mathrm{~K}$ (Methods section). The corresponding ARUPS intensity 
a

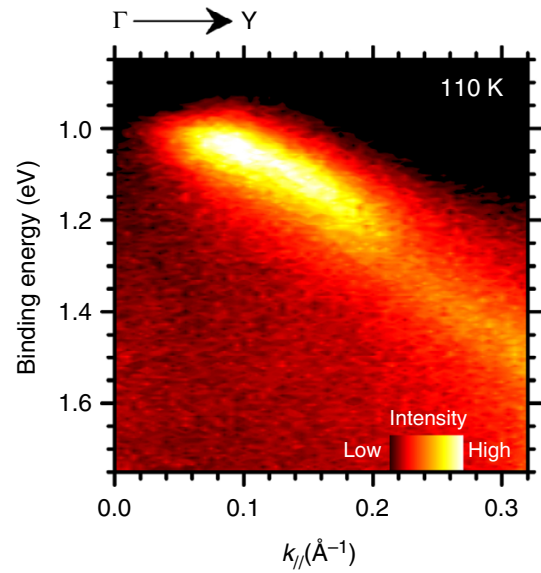

b

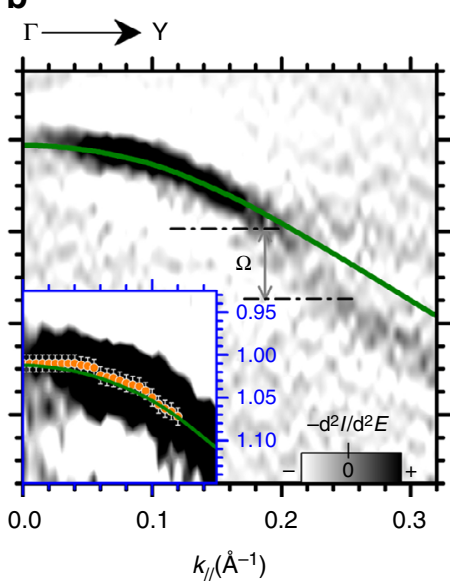

c

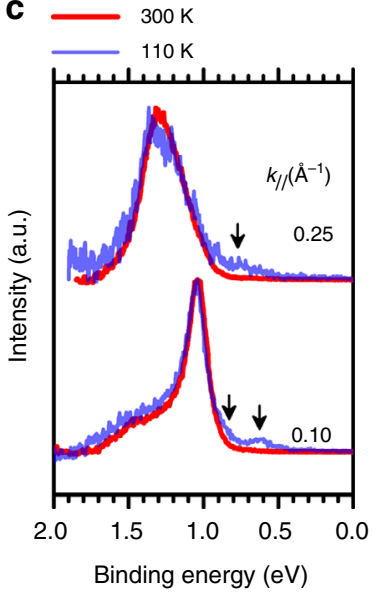

Fig. 3 Angle resolved band map at $110 \mathrm{~K}$ and theoretical calculations. a ARUPS intensities vs. $k / /$ along the $\Gamma Y$ direction of rubrene single crystal at $110 \mathrm{~K}$; b Second derivative ARUPS intensity map $\left(-d^{2} l / d^{2} E\right.$, where $I$ is the ARPES intensity and $E$ the binding energy) as obtained from data in $\mathbf{a}$. The splitting of the $\mathrm{HOMO}$ band in two subband $\mathrm{H}^{\mathrm{U}}$ and $\mathrm{H}^{\mathrm{L}}$ is evidenced as separated by gap $\Omega$. Inset: Magnification of second derivative map close to $\Gamma$ point.

Experimental $\mathrm{H}$ peak positions are also indicated by orange circles and compared with theoretical band dispersion (continuous green line) $\mathbf{c} \mathrm{HOMO}$-EDC curves (Shirley background subtracted, intensity normalized at the curve maximum) at $k_{/ /}=0.10 \AA^{-1}$ and $k_{/ /}=0.250 \AA^{-1}$, as obtained at $300 \mathrm{~K}$ (red line) and $110 \mathrm{~K}$ (blue line). Peak like/tailing structure (arrow) are related to structural defects resulting from the temperature-induced mechanical stress in the rubrene single crystal
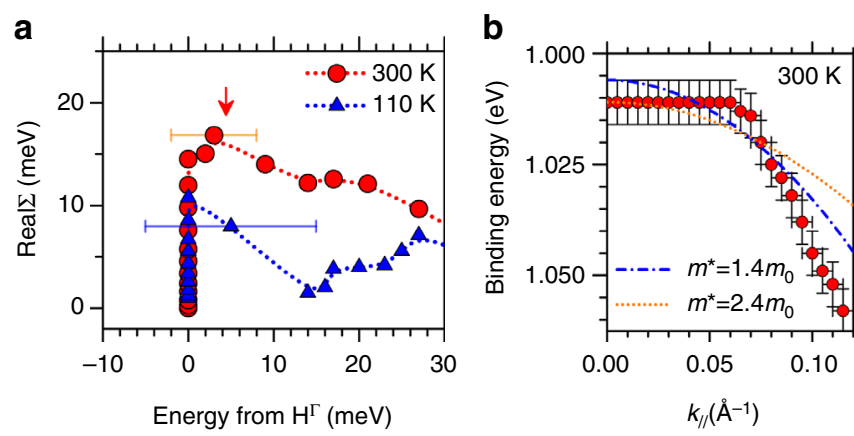

Fig. 4 Real part of Self Energy and hole effective mass. a Real part of quasiparticle self-energy $(\Sigma)$ at 300 and $110 \mathrm{~K}$ (see inset in Figs $2 \mathrm{~b}$ and $3 b$ ). The energy values were evaluated with respect to the $\mathrm{H}$ peak position at $\Gamma$ point $\left(\mathrm{H}^{\Gamma}\right.$ ), the error bar being $\pm 5 \mathrm{meV}$ (at $300 \mathrm{~K}$ ) and $\pm 10 \mathrm{meV}$ (at $110 \mathrm{~K}$ ) as resulting from the EDCs peak fitting procedure $\mathbf{b}$ Experimental $\mathrm{H}$ band dispersion near $\Gamma$ point region at $300 \mathrm{~K}$, with corresponding energy error bars $( \pm 5 \mathrm{meV})$. The band curvature close to $\Gamma$ point was estimated by means of parabolic curves centered at $\Gamma$ within the $[0-0.07] \AA^{-1}$ wave vector range, where kink is observed, and corresponding effective mass value was extracted

map and the results of second derivative analysis are plotted in Fig. 3a, b, respectively. At low temperature the kink-like feature near $\Gamma$ point is no longer visible, with the curvature of the $\mathrm{H}$ band well reproduced by the theoretical calculations. With respect to $300 \mathrm{~K}$ measurements, no significant changes in the energy gap size and position were detected, as well in the energy width of the HOMO-EDCs (Fig. 3c). A comparison between the EDCs as obtained at 300 and $110 \mathrm{~K}$ in the $[0 \sim 0.095] \AA^{-1}$ wave vector range is shown in Supplementary Fig. 5 to illustrate the temperature-induced binding energy shift resulting in the quenching of the kink structure.

Gap opening in the HOMO band dispersion. The impact of the molecular vibrations on the electronic properties of organic materials was experimentally investigated by ARUPS for thin films of weakly interacting molecules ${ }^{19}$ where non-dispersive HOMO bands consisting of several components with comparable energy separations were observed ${ }^{19}$. Under simplified assumptions (single mode analysis), this energy separation $\left(\Omega_{0}\right.$, typically $\gtrsim 100 \mathrm{meV}$ ) corresponds to the average intramolecular vibration (local hole-phonon coupling effect) energy while the relative intensities of the HOMO components reflects the coupling strength $^{19}$. This approach was theoretically generalized to the case of molecular crystals where larger HOMO bandwidths $(W)$ are expected ${ }^{11}$. When $W \gtrsim \Omega_{0}$, the vibrational structure characteristic of the thin film photoemission spectra is replaced by "cuts" in the energy band dispersion. Spectral intensity modulation is thus introduced along the energy axis and gap-like structures appears across the HOMO band dispersion ${ }^{11}$. The energy gap size and position in the energy/momentum space reflect the value of the intramolecular vibration energies and the strength of the corresponding hole-phonon coupling, as discussed in the theorethical background of Supplementary Note 3. In this context, the gap-like feature in $\mathrm{H}$ band dispersion (bandwidth $\sim 0.52 \mathrm{eV}$ ) of rubrene single crystal (Fig. 2a, b) can be related to the hole coupling with intramolecular vibrations with an average energy of $\sim 140 \mathrm{meV}$. An average intramolecular vibrational energy of $\sim 130 \mathrm{meV}$ was extracted by the single mode analysis of ARUPS-HOMO line shape for rubrene thin film, in good consistence with the present result ${ }^{20}$.

The local nature of the hole-phonon coupling process explains the rapid change in the $\mathrm{H}$ peak width (and related hole lifetime) upon gap crossing along the $\Gamma Y$ direction (Fig. 2c). When a hole is created in the $\mathrm{H}^{\mathrm{U}}$ band, the electrons at lower binding energies cannot be scattered into the empty electronic states by intramolecular phonon activation process, as the energy difference between the initial and final electron states is lower than the intramolecular vibration energy $(\sim 140 \mathrm{meV})$. The suppression of the scattering mechanism results in a large hole lifetime. The situation is reversed in the lower $\mathrm{H}^{\mathrm{L}}$ band where the electron scattering as mediated by intramolecular phonon activation is energetically possible, thus leading to decreasing of the hole lifetime.

Finally, as shown in Fig. 3 the gap size and $\mathrm{H}$ peak width are not affected by the sample temperature. In (110-300 K) range, in 
fact, the thermal energy $k T(<25 \mathrm{meV})$ is much lower with respect to the rubrene intramolecular vibrational energy $(\sim 140 \mathrm{meV})$. Therefore no intramolecular vibrations can be thermally excited/quenched and their impact of the band dispersion and hole lifetime is thus largely temperature independent as confirmed by the experimental data.

Kink structure in HOMO band dispersion. Kink-like structures were experimentally observed in the ARUPS measurements of metal conduction band and related to the hole coupling with the lattice vibrations ${ }^{9}$. The position of the kink with respect to Fermi level (i.e., the highest occupied level for metal) corresponds to the energy of the coupled vibrational mode ${ }^{9}$.

In analogy with the inorganic case, the kink structure near the top of the rubrene $\mathrm{HOMO}$ band $(<20 \mathrm{meV})$ at $300 \mathrm{~K}$ (inset Fig. 2b) can be related to the hole coupling with low energy intermolecular vibrations (non-local hole-phonon coupling) of rubrene single crystal.

From a careful comparison between the experimental data and theoretical calculations near the $\Gamma$ point (see Methods section) the real part $(\operatorname{Real} \Sigma)$ of the hole quasiparticle self-energy $(\Sigma)$ was extracted. The results of the analysis are reported in Fig. 4a. At $300 \mathrm{~K}$, a peak at $\sim 8 \mathrm{meV}$ from the top of the HOMO band $(\mathrm{H \Gamma )}$ is visible. According to the general theory of photoemission spectral function 9,16 the position of the peak in the real part (Real $\Sigma)$ of the quasiparticle spectral function corresponds to the energy of the (intermolecular) phonon involved in the hole-vibration coupling 9,16 . Strongly coupled intermolecular vibrational modes were predicted at $4-16 \mathrm{meV}$ by phonon spectra calculation of rubrene single $\mathrm{crystal}^{8}$, in good agreement with the present findings.

At $110 \mathrm{~K}$ no peak like feature is observed in the extracted Real $\Sigma$ (Fig. 4a), which corresponds to the disappearance of the kink-like structure in the low temperature ARUPS data (inset of Fig. 3b). The low energy intermolecular vibrations are expected to be rapidly quenched by the temperature reduction as their energies is comparable with $k T$ value in $[110-300 \mathrm{~K}]$ temperature range $(<25 \mathrm{meV})$. In this context, the temperature dependent ARUPS study supports the local nature of the hole-coupling effect near the top of the rubrene HOMO band.

\section{Discussion}

Our ARUPS investigation can help to clarify important aspects of the hole dynamics in rubrene single crystal, which are crucial for the charge transport mechanism. As previously discussed, the hole lifetime in the upper $\mathrm{H}^{\mathrm{U}}$ band (originating from local hole-phonon coupling effect) is not affected by the scattering with intramolecular phonons (Fig. 2c). Since only the hole states near the HOMO band edges can be thermally populated or doped in any real organic-based device ${ }^{15}$, it is possible to conclude that the scattering from high energy intramolecular modes does not play a significant role in the observed charge transport properties of rubrene single crystal.

Near the top of the HOMO band, the non-local hole-phonon coupling leads to a change (i.e., decrease) of the band curvature $\mathrm{d}^{2} E\left(k_{/ /}\right) / \mathrm{d}^{2} k_{/ /}$with respect to the results of the theoretical calculation. This directly affects the value of the hole effective mass $m^{*}\left(\right.$ as $m^{*} \propto 1 /\left(\mathrm{d}^{2} E\left(k_{/ /}\right) / \mathrm{d}^{2} k_{/ /}\right)^{4}$ which is an essential parameter to define the charge mobility $(\mu)$ in both inorganic and organic systems ${ }^{4,9}$. The band curvature was measured by parabolic fitting of the experimental HOMO dispersion near the $\Gamma$ point, as shown is Fig. $4 \mathrm{~b}$ (see Methods section). A value of $m^{*}>1.4 m_{0}$ was obtained (where $m_{0}$ is the hole mass at rest) which is well above the theoretical value $\left(0.8 \sim 0.9 m_{0}\right)$ as estimated on the basis of band structure calculations and commonly used to in charge transport model of rubrene single crystal $^{10,15,18}$.

Despite the conceptual limit of the band model discussed in the introduction, the increase of the hole effective mass $\left(m^{*}>1.4 m_{0}\right)$ rationalizes quite well the theoretical overestimation of the rubrene hole mobility with respect to the experimental value, as obtained by band-like transport model where $\mu \propto 1 / \mathrm{m}^{*}$ with $m^{*}=0.8 \sim 0.9 m_{0}{ }^{10}, 15,18$. The effective mass enhancement quantitatively describes the hole localization process as related to the charge coupling with intramolecular vibrations of rubrene lattice ${ }^{8}$.

The hole localization effects in rubrene single crystal are basically quenched as $110 \mathrm{~K}$, where the band dispersion near the HOMO edge positions are well described by theoretical calculations (Fig. 3b). This result is consistent to the recovery of band transport limit and the increase of the intrinsic hole mobility at low temperature, as reported for rubrene and other organic crystalline system ${ }^{13}$.

In the present research, we report a rigorous experimental investigation on the electronic properties of rubrene single crystal, a prototypical example of organic semiconductor. We provided a clear evidence of peculiar features in the band dispersion which are not predicted by usual theoretical band structure calculations. In analogy with ARUPS theory, this deviation strongly resembles the effect of charge-phonon coupling in one-hole state as commonly reported in ARUPS investigation of inorganic single crystal. The temperature dependent data supported this interpretation. Moreover this is also consistent with the current state of the art of hole quasiparticle spectral function calculation in organic systems, with obvious deviations as related to the difference between our investigated system and the simplified assumption in the theoretical models. We also provide direct evidence on how charge-phonon coupling may increase charge localization, well above the limit predicted by DFT band structure calculation, which is a relevant result for applications. A complete simulation of the quasiparticle spectral function with theoretical calculation of the UPS spectra as well as a more quantitative modeling of charge transport in organic materials will help in a full rationalization of the present experimental results. In this context, the present work can attract interest of theoretical groups and stimulate the theoretical debate thus representing an important step towards the full understanding of the impacts of charge-phonon coupling on the electronic and transport properties of organic semiconductor crystals.

\section{Methods}

Synthesis of single crystal and sample preparation. Rubrene single crystals were prepared by physical vapor transport technique under nitrogen stream. Elongated sample of similar lateral size $(\sim 2 \times 5 \mathrm{~mm})$ and thickness $(\sim 200 \mu \mathrm{m})$ were mounted on a conductive carbon tape (STR-9181, Shinto Chemitron Co., LTD) positioned on a copper sample holder. Then, the edges of the single crystals were carefully covered with conductive carbon paste in order to improve the mechanical and electric contact with the underlying carbon tape.

ARUPS measurements. ARUPS study was conducted at beamline BL7U of UVSOR synchrotron facility (Okazaki, Japan) according to the experimental geometry described in Fig. 1c. For all the investigated samples, comparable photoemission results were found. During the photoemission the sample were concurrently irradiated with continuous laser light (energy $=3.06 \mathrm{eV}$, power 10 $\mathrm{mW}$ ) to overcome the sample charging effects induced by the ionizing UV radiation due to the high electrical resistance of organic single crystals. Under the present experimental conditions the sample charging was completely quenched as evaluated by preliminary ARUPS investigations and in agreement with previous experimental reports ${ }^{21}$

In some of the prepared rubrene single crystals, the sample charging induced by the UV light irradiation was found to be considerably reduced, probably due to a better crystal quality and lower sample thickness. In these cases, a fast ARUPS data acquisition was also possible without concomitant laser light irradiation. These data were compared with ARUPS data obtained under laser light irradiation (not shown). The comparison indicates the absence of any significant effect of the 
laser light irradiation on the key features (i.e., kink, bandwidth, and so on) of the measured band dispersion of rubrene single crystal.

The UV light spot size on the sample was $\sim 1 \mathrm{~mm} \times 100 \mu \mathrm{m}$. Electron emission angle $\theta$ was measured with respect to the normal to the sample surface and photoelectrons were collected in the $\left(-6^{\circ} ;+30^{\circ}\right)$ emission angular range (Fig. 1c) at the angular resolution of $\sim 0.16^{\circ}$. The binding energy value was referenced to the Fermi Level $\left(E_{\mathrm{F}}\right)$ measured on a polycrystalline gold sample. The energy resolution was better than $10 \mathrm{meV}$ as judged from the Fermi edge of the reference gold sample. The rubrene single crystals were oriented according to the anisotropy of crystal shape which reflects the symmetry of the crystal unit cell in real and reciprocal space ${ }^{21}$. All samples were measured as-inserted i.e., no sample cleaving was performed after its introduction into UHV environment. In the investigated photoelectron kinetic energy range $\left(E_{\text {kin }}<8 \mathrm{eV}\right)$ the estimated electron mean free path is $\sim 3 \mathrm{~nm}^{22}$ which corresponds to several rubrene layers. The ARUPS data therefore largely reflect the electronic properties of the bulk region and are rather insensitive to the structural and chemical defects of the surface region due to gaseous contamination and/or photo oxidation process $^{23}$.

For temperature dependent ARUPS study the sample was slowly cooled $(\sim 18 \mathrm{~h})$ from 300 to $110 \mathrm{~K}$. The cooling rate was kept slow in order to minimize temperature-induced mechanical stress in the crystal and at the contact between sample and conductive paste/tape. Temperature-induced defects in the single crystal resulted in localized states in the rubrene energy gap as shown by the peak like and tailing feature in the low temperature HOMO lineshape $\mathrm{e}^{21}$

During the low temperature ARUPS measurement, the incident photon flux was $\sim 4$ times reduced to compensate the decrease of the electrical conductivity of carbon paste and tape at low temperature and avoid sample charging.

EDCs fitting analysis. The HOMO-EDCs at various $k_{/ /}$was analyzed by least square fitting procedure. The $\mathrm{H}$ peak was simulated by Voigt function. The FWHM of the Gaussian component $w_{\mathrm{G}}$ was set within [130 150] meV to reproduce the Gaussian broadening which originates from (i) relaxation/polarization effect resulting from structural disorder and (ii) experimental resolution ${ }^{19}$. The A and $\mathrm{B}$ features was simulated by superimposition of Gaussian peaks. Representative fitting results of EDCs at various $k_{/ /}$are reported in Supplementary Fig. 6.

In obtaining the plot of Fig. $2 \mathrm{c}$, for each $k_{/ /}$a Lorentzian curve centered at binding energy $x_{\mathrm{C}}$, with width $w_{\mathrm{L}}$ and initially arbitrary intensity at peak maximum (A) was plotted. The intensity at peak maximum were adjusted to results in the same integrated area for each Lorentzian function, the integral being numerically evaluated in the $\left(x_{\mathrm{C}}-5 \cdot w_{\mathrm{L}} ; x_{\mathrm{C}}+5 \cdot w_{\mathrm{L}}\right)$ energy range. For the gap region where two Lorentzian curves were extracted, the superimposition of the two curves was considered. The integral were calculated in the $\left(x_{\mathrm{C}}^{\mathrm{UP}}-5 \cdot w_{\mathrm{L}}^{\mathrm{UP}} ; x_{\mathrm{C}}^{\mathrm{LO}}+5 \cdot w_{\mathrm{L}}^{\mathrm{LO}}\right)$ binding energy range where $x_{\mathrm{C}}^{\mathrm{Up}}\left(x_{\mathrm{C}}^{\mathrm{Lo}}\right)$ and $w_{\mathrm{L}}^{\mathrm{Up}}\left(w_{\mathrm{L}}^{\mathrm{Lo}}\right)$ are the peak center (Lorentzian width) of $\mathrm{H}^{\mathrm{U}}$ and $\mathrm{H}^{\mathrm{L}}$ band components (Fig. $2 \mathrm{~b}$ ).

Self-energy analysis. The self-energy $\Sigma$ was defined as in ref. ${ }^{16}$ i.e., $A\left(k_{/ /}, E\right) \propto$ $\operatorname{Im} \Sigma(E) /\left(\left[E-\varepsilon_{k}-\operatorname{Real} \Sigma(E)\right]^{2}+\left[\operatorname{Im} \Sigma(E)^{2}\right]\right)$ where $A\left(k_{/ /}, E\right)$ is the quasiparticle spectral function, $\varepsilon_{k}$ the non-interacting band dispersion. The real part Real $\Sigma$ of the quasiparticle self-energy was extracted from HOMO-EDCs at various $k_{/ /}$by evaluating the differences between experimental and theoretical peak position. In standard ARUPS analysis, this quantity is generally extracted by comparing experimental and theoretical momentum distribution curves (MDCs). In the present case, however, the reduced HOMO band dispersion near $\Gamma$ point $(<10 \mathrm{meV})$ introduces large $k_{/ /}$broadening of the MDCs curve and large uncertain in the experimental HOMO peak position. The same reasons hinder a direct evaluation of the imaginary part $\operatorname{Im} \Sigma$ of the quasiparticle self-energy which reflects the $k_{/ /}$broadening of the experimental MDCs curves ${ }^{16}$

\section{Estimation of the hole effective mass. The hole effective mass at HOMO} edge was defined as $\left.m^{*}=\hbar^{2} /\left[\mathrm{d}^{2} \mathrm{E}\left(\mathrm{k}_{/ / /}\right) / \mathrm{d}^{2} k_{/ /(k / /=0))}\right)\right]$, where $\mathrm{d}^{2} \mathrm{E}\left(\mathrm{k}_{/ /}\right) / \mathrm{d}^{2} k_{/ /(k / /=0)}$ is the band curvature at $\Gamma$ point $\left(k_{/ /}=0.000 \AA^{-1}\right)$. The band curvature was measured by parabolic fitting of the experimental data in the $[0.000-0.070] \AA^{-1}$ wave vector range, which reflects the band distortion produced by the kink. A value of $1.4 m_{0}<m^{*}<2.4 m_{0}$ was found, considering the binding energy and wave vector uncertain in the HOMO peak positions. Representative parabolic fitting curves for the $m^{*}$ upper and lower limit are reported in Fig. 2b. The same fitting method was applied to the theoretical band dispersion ${ }^{18}$ within the same wave vector range. A value of $m^{*}=0.9 m_{0}$ was extracted which is consistent with the theoretical estimation in ref. ${ }^{18}\left(0.9 \mathrm{~m}_{0}\right)$ and in good agreement with previous reports $\left(\sim 0.8 m_{0}\right)^{10,15}$

Data availability. The data that support the findings of this study are available from the corresponding authors upon reasonable request.

Received: 23 January 2017 Accepted: 13 June 2017

Published online: 02 August 2017

\section{References}

1. Dimitrakopoulos, C. D., Purushothaman, S., Kymissis, J., Callegari, A. \& Shaw, J. M. Low-Voltage organic transistors on plastic comprising highdielectric constant gate insulators. Science 283, 822-824 (1999).

2. Reineke, S. et al. White organic light-emitting diodes with fluorescent tube efficiency. Nature 459, 234-238 (2009).

3. Uoyama, H., Goushi, K., Shizu, K., Nomura, H. \& Adachi, C. Highly efficient organic light-emitting diodes from delayed fluorescence. Nature 492, 234-238 (2013).

4. Ueno, N. \& Kera, S. Electron spectroscopy of functional organic thin films: deep insights into valence electronic structure in relation to charge transport property. Prog. Surf. Sci. 83, 490-557 (2008).

5. Mitsutake, K. \& Tsukada, M. Theoretical study of electron-vibration coupling on carrier transfer in molecular bridges. Surf. Sci. Nanotech. $\mathbf{4}$ 311-318 (2006).

6. Fratini, S., Mayou, D. \& Ciuchi, S. The transient localization scenario for charge transport in crystalline organic materials. Adv. Funct. Mater. 26, 2292-2315 (2016).

7. Troisi, A. \& Orlandi, G. Dynamics of the intermolecular transfer integral in crystalline organic semiconductors. J. Phys. Chem. A 110, 4065-4070 (2006).

8. Girlando, A. et al. Peirls and Holstein carrier phonon coupling in crystalline rubrene. Phys. Rev. B 82, 035208 (2010).

9. Plummer, E. W., Shi, J., Tang, S. J., Rotenberg, E. \& Kevan, S. D. Enhanced electron-phonon coupling at metal surfaces. Prog. Surf. Sci. 74, 251-268 (2003)

10. Li, Y., Yi, Y., Coropceanu, V. \& Bredas, J. L. Symmetry effects on nonlocal electron-phonon coupling in organic semiconductors. Phys. Rev. B 85, 245201 (2012).

11. Ciuchi, S. \& Fratini, S. Band dispersion and electronic lifetimes in crystalline organic semiconductors. Phys. Rev. Lett. 106, 166403-166406 (2011).

12. Takeya, J. et al. Very high-mobility organic single-crystal transistors with in-crystal conduction channels. Appl. Phys. Lett. 90, 102120-101122 (2007).

13. Coropceanu, V. et al. Charge transport in organic semiconductors. Chem. Rev. 107, 926-952 (2007).

14. Reese, C. \& Bao, Z. High resolution measurements of the anisotropy of charge transport in single crystals. Adv. Mater. 19, 4535-4538 (2007).

15. Podzorov, V. et al. Intrinsic charge transport on the surface of organic semiconductors. Phys. Rev. Lett. 93, 086602-086604 (2004).

16. Damascelli, A., Hussain, Z. \& Shen, Z.-X. Angle-resolved photoemission studies of the cuprate superconductors. Rev. Mod. Phys. 75, 473-541 (2003).

17. Lindroos, M., Sahrakorpi, S. \& Bansil, A. Matrix element effects in angleresolved photoemission from $\mathrm{Bi}_{2} \mathrm{Sr}_{2} \mathrm{CaCu}_{2} \mathrm{O}_{8}$ : energy and polarization dependencies, final state spectrum, spectral signatures of specific transitions, and related issues. Phys. Rev. B 65, 054514-054525 (2002).

18. Yanagisawa, S., Morikawa, Y. \& Schindlmayr, A. HOMO band dispersion of crystalline rubrene: effects of self-energy corrections within the GW approximation. Phys. Rev. B 88, 115438-115444 (2013).

19. Kera, S., Yamane, Y. \& Ueno, N. First-principles measurements of charge mobility in organic semiconductors: valence hole-vibration coupling in organic ultrathin films. Prog. Surf. Sci. 84, 135-154 (2009).

20. Duhm, S. et al. Charge reorganization energy and small polaron binding energy of rubrene thin films by ultraviolet photoelectron spectroscopy. Adv. Mater. 24 901-905 (2012)

21. Machida, S. I. et al. Highest-occupied-molecular-orbital band dispersion of rubrene single crystals as observed by angle-resolved ultraviolet photoelectron spectroscopy. Phys. Rev. Lett. 104, 156401-156404 (2010).

22. Seah, M. P. \& Dench, W. A. Quantitative electron spectroscopy of surfaces: A standard data base for electron inelastic mean free paths in solids. Surf. Interface Anal. 1, 1:2-11 (1979).

23. Mastrogiovanni, D. D. T. et al. Oxygen incorporation in rubrene single crystals. Sci. Rep. 4, 4753-4758 (2014).

\section{Acknowledgements}

The authors thank UVSOR staff members for experimental support. The study is partly supported by JSPS KAKENHI (nos. 26248062, 23360005, 15H05498), and Global COE program at Chiba University (G-03, MEXT). Y.J. acknowledges the support from NSFC (No. 61504119), Natural Science Foundation of Jiangsu Province, China (BK20150458).

\section{Author contributions}

The experimental data were measured by F.B., J.Y., T.Y., Y.N., K.Y., K.S. and S.K. and analyzed by F.B.; Y.N. synthesized the samples used in this study; K.T. and M.M. maintained the synchrotron ARUPS end stations and provided experimental support; F.B. wrote the manuscript, with input and discussions from all coauthors; H.I., S.K. and 
N.U., conceived the study and were responsible for overall project planning and direction.

\section{Additional information}

Supplementary Information accompanies this paper at doi:10.1038/s41467-017-00241-z.

Competing interests: The authors declare no competing financial interests.

Reprints and permission information is available online at http://npg.nature.com/ reprintsandpermissions/

Publisher's note: Springer Nature remains neutral with regard to jurisdictional claims in published maps and institutional affiliations. (c) Open Access This article is licensed under a Creative Commons Attribution 4.0 International License, which permits use, sharing, adaptation, distribution and reproduction in any medium or format, as long as you give appropriate credit to the original author(s) and the source, provide a link to the Creative Commons license, and indicate if changes were made. The images or other third party material in this article are included in the article's Creative Commons license, unless indicated otherwise in a credit line to the material. If material is not included in the article's Creative Commons license and your intended use is not permitted by statutory regulation or exceeds the permitted use, you will need to obtain permission directly from the copyright holder. To view a copy of this license, visit http://creativecommons.org/ licenses/by/4.0/.

(C) The Author(s) 2017 